\title{
The Application of CFD in Teaching of Fluid Machinery*
}

\author{
Zhiqiang Wang \\ School of Mechanical Engineering \\ Hangzhou Dianzi University \\ Hangzhou, China
}

\author{
Dianrong Gao \\ College of Mechanical Engineering \\ Yanshan University \\ Qinhuangdao, China
}

\begin{abstract}
Aiming at the characteristics of the course of Fluid Machinery and the weakness of the traditional teaching, CFD technique was introduced into classroom teaching and the fluid components were simulated by applying the CFD software. CFD technique can record and reconstruct the whole flow information of the fluid components, and may realize visualization in virtual reality really. The application of CFD technique can not only arouse the students' interests and build up sensibility cognition of students, make the teaching change from theoretical and principle mode to liberal and application mode, but also help students to understand and master the design of the internal construction of fluid components and the meanings of the parameters. Finally, this application realizes the expansion of teaching content and results in an elevation of teaching quality.
\end{abstract}

Keywords-CFD technique; fluid Machinery; blood pump; stirred tank

\section{INTRODUCTION}

Going with the rapid development of computer technologies and the continuous improvement of numerical simulation precision and reliability, CFD simulation analysis has become one of the main methods for the fluid analysis and the role it plays is increasingly highlighting in fluid machinery, hydraulic components and pipeline dynamics. For the CFD simulations have characteristics such as high applicability, wide application and no limitation in physical and experimental models, scholars at home and abroad have conducted deep researches on centrifugal pumps, axial pumps, piston pumps and gear pumps through the CFD technology.

In order to extract the dipole source of the wall of volute and leakage flow, Tan Minggao et al selects a single-stage single-suction vertical centrifugal pump with six blades with the whole flow field numerical simulation executed by applying CFD[1]. Zhou Linyun adopts large eddy simulation and sliding mesh technique to analyze the pressure fluctuation and vibration of the centrifugal pump under deviation, discovering that in most cases, the pressure fluctuation produced by the flow inside the centrifugal pump is its passing frequency[2]. Zhang Chixiang et al adopt RNG $\kappa-\varepsilon$ turbulence model and SIMPLEC algorithm to conduct researches on characteristics of flows inside the centrifugal pump under extremely high flow[3]. Liu et al adopts CFD

*The research subjects of higher education of Zhejiang Province (kg20160133) simulation toolbox to directly simulate the flow inside the pumps with double blades under a steady condition [4]. Yang Guolai et al adopt dynamic mesh technology in the CFD to conduct dynamic numerical simulation and analysis on the valve flap movement of the axial flow check valve and the flow state of the cone valve [5], [6]. Blanco et al adopt dynamic mesh technologies to conduct dynamic numerical simulation on the pneumatic piston pump [7]. Castilla et al adopt dynamic mesh technologies to conduct dynamic numerical simulation on changes of flow fields inside the gear pump [8].

Through the CFD simulation technology, we can get the distribution of pressure, speed, temperature and so on in the fluid model as well as their changes varying with the time, besides, and the parameters can be set as required to meet the harsh conditions that are hard to be achieved in real conditions. Therefore, during the teaching of fluid machinery, in order to enable students to understand and clearly see the changes of the fluid in the components, the CFD technology can be adopted to simulate the internal flow field and to reproduce the flow of the flow field, achieving a real visualization, which can not only stimulate students' interests to study but also help them understand and grasp the design of internal structures of components as well as the meaning of the parameters.

\section{APPLICATION OF THE CFD IN TEACHING}

\section{A. Tampered Axial Blood Pump}

Axial blood pump is one of the impeller pumps of fluid machinery, the pump works relying on the impeller rotating the blood, transmitting the mechanical energy to the blood so as to achieve the blood transport. Compared to ordinary impeller pumps, the pump works in a blood system of human body, so it has specific requirements on pressure intensity and flow. In this example, the pump output flow is required to reach 4 6L / min, with a pressure intensity of 120 140 $\mathrm{mmHg}$.

How does the blood flow inside the axial blood pump? How does its structure affect the blood flow? These problems can not be solved only by the information given in textbooks. If a prototype is used for testing, its cost is too high. At this time the CFD can be used to simulate the flow field inside the blood pump to visualize the flow field. 
The CFD simulation will require modeling, mesh generation, boundary conditions setting as well as calculation of the final flow field. In this case, the flow field diagram inside the blood pump, mesh generation diagram and boundary condition setting are shown in "Fig. 1", "Fig. 2" and "Fig. 3".

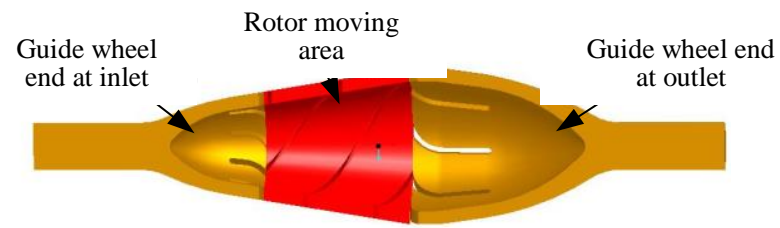

Fig. 1. Blood flow field area.

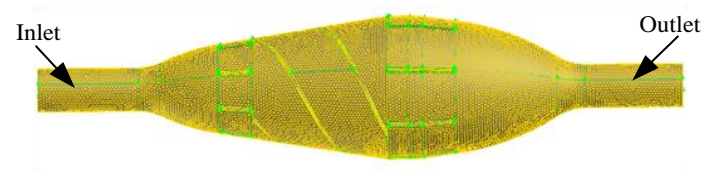

Fig. 2. Blood pump mesh generation model.

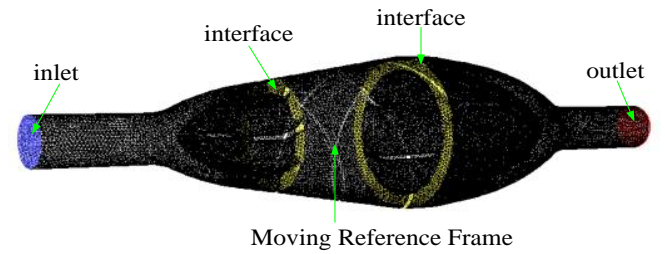

Fig. 3. Setting of boundary conditions.

After completion of the mesh generation, set the boundary type, for the fluid flows from left to right inside the pump, so set the left as the pressure inlet, the right as the pressure outlet, as shown in "Fig. 2". At the same time, for the rotor inside the blood pump is a rotating unit, so the fluid inside it shall be set to Moving Reference Frame, and set the part contacting the rotor to be Moving wall - rotational, and connect with the front and rear guide wheel areas through the interface so as to ensure the data transfer and exchange, as shown in "Fig. 3".

With both the flow and pressure intensity of the blood pump met, conduct numerical simulation on the flow filed of the blood pump, and the blood flow inside can be seen via the flow path of the blood pump so as to further improve the structure. Here is analysis of rotor's blades on the flow, "Fig. 4" flow traces inside the blood pump.

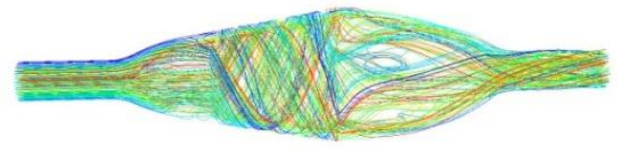

a) Traces of rotor with 2 blades.

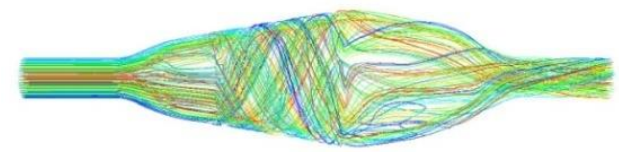

b) Traces of rotor with 3 blades.

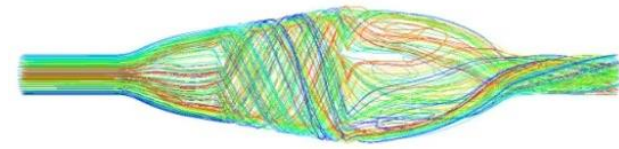

c) Traces of rotor with 4 blades.

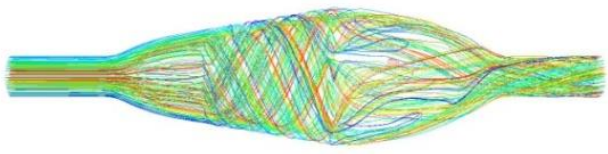

d) Traces of rotor with 5 blades.

Fig. 4. Internal traces of blood pump with different rotations.

Seen from "Fig. 4", on the front wheel, the blood pump flow line with 2, 3 rotor blades shows a slight return and turbulence, while the blood pump with 4,5 rotor blades shows better flow traces. When the fluid enters the rear guide wheel area through the helical impeller area in the middle, it can be seen that four types of rear wheel areas all have significant backflow and flow disturbance, besides, the fluid at the outlet rotates, which is not good to the blood flow in the body. The rotating fluid may cause the blood at the output to produce tangential components, due to the effect of tangential shear, the blood cell will rupture, and that is, hemolysis appears.

\section{B. Dual Impeller Stirred Tank- Example 2}

Stirred tank is designed with a simple structure, belonging to the fluid machinery, for it is easy to control and runs steadily, it has been widely used in construction, chemical industry, food processing, sewage treatment and so on. Main indices for evaluating the performance of the stirred tank are uniformity of co-blending and blending time. The uniformity of co-blending means during the operation, whether the stirred tank can finally mix two or more media inside it into a wellmixed material. The blending time refers to the time needed from initial blending to one medium by the co-blended media. During the co-blending, that is, during the flow and diffusion of the media, how the process is formed cannot be shown by formulas in textbooks, and also unable to be described vividly. Now it is time for the CFD simulation, which can show the flow and diffusion of the media in the stirred tank.

Refer to "Fig. 5" for the structure of dual -impeller stirred tank, refer to "Fig. 6" for the mesh generation, seen from the figures, the stirred tank is divided into the area of upper paddle, the area of lower paddle and the area between the two paddles.

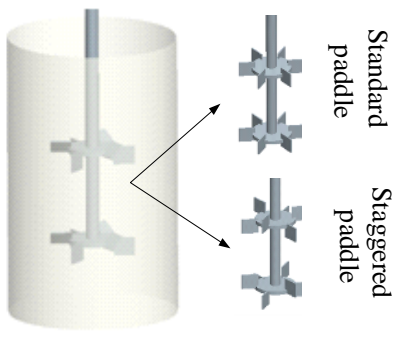

Fig. 5. Dual impeller stirred tank. 


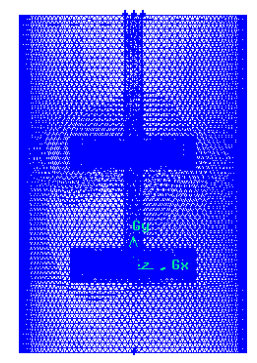

Fig. 6. Stirred tank mesh generation.

In order to enable students to clearly watch the changes of flow fields when the media are stirred, take the vertical surface of the stirred tank as an example, observe the speed changes of the media on the vertical surface, of which, the media stirred shall adopt the mixture of glycerol and water, having a density of $1250 \mathrm{~kg} / \mathrm{m} 3$ and a kinematic viscosity of 0.4 Pa.s. "Fig. 7", "Fig. 8", "Fig. 9", "Fig. 10" show the velocity vector and velocity cloud diagram of the blended media on the vertical surface for different paddle structures.

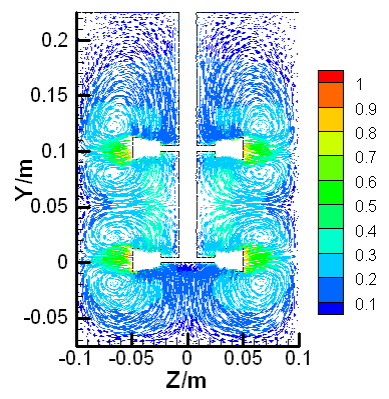

Fig. 7. Standard paddle viscosity vector.

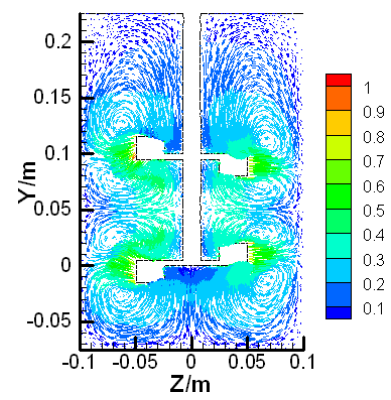

Fig. 8. Staggered paddle viscosity vector.

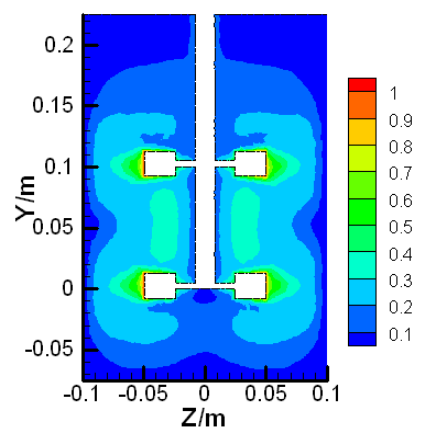

Fig. 9. Standard paddle speed cloud.

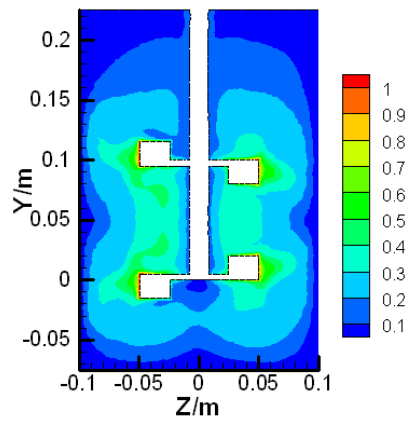

Fig. 10. Staggered paddle speed cloud.

Seen from Fig 7 to Fig 10, whether the standard paddle or the staggered paddle, its changes of flow field takes on symmetric distribution along the paddle shaft, yet the standard paddle forms uniform eddies around the paddle, while the staggered paddle also forms eddies on the below the paddle, but different in the size of eddies, and the diameter of the eddy outside the paddle is larger than that of the eddies in the middle. Besides, we can see that the two paddles both exclude the area with an extremely low speed value under the lower paddle, namely this part will be a dead area, which is free from the effect of the paddles, and the media in the area can flow freely only depending on themselves, but the dead areas of two paddles differ, and the dead area of the staggered paddle is much smaller than that of the standard paddle. Seen from Fig 9 and Fig 10, the high-speed area of the staggered paddle is larger than that of the standard one, which turns out that that the staggered distribution of paddles enhances the velocity of the media in the stirred tank, increasing the highspeed area for co-blending, doing good to the mixture of a variety of media.

Seen from the analysis results of the velocity vector and the velocity cloud diagram in Fig 7 to Fig 10, whether the standard paddle or the staggered paddle, when it rotates, the centrifugal force will promote all fluids in the tank to flow, yet the flow speed of liquids at different locations will differ in the tank, at this time, the high-speed fluid and the lowspeed fluid will form eddies when moving due to the shearing of liquid because of the speed gradient. At the same time, for the maximum speed occurs where mixed media contact outside the paddles, so the movement there will be the most intense. When the blended liquid is pushed to the wall, it will be divided into two streams running along the wall up and down so as to form two large eddies.

Seen from the two examples mentioned above, the CFD technology can be used to analyze fluid components, as long as the boundary conditions are given, the parameters can be changed for different test results, which well solves the problems such as long test period, high costs and difficult conditions, and the students can directly watch the flow trace, velocity field and pressure field of the fluid inside components.

\section{CONCLUSION}

In conclusion, during the lecture of fluid machinery, the use of CFD technology can not only visually show the 
changes of flow fields inside the flow components under different test conditions but also make students rapidly master the states of flow machinery in operation, improving students' perceptual knowledge and widening their thoughts, so that the teaching will be changed to application and comprehension from principles, it will do good to benefit the students, expand teaching contents and improve the teaching efficiency.

\section{REFERENCES}

[1] Tan Minggao,Zhang Jing, Liu Houlin, etal. Effect of uneven spacing blade on hydrodynamic noise of centrifugal pump[J]. Transactions of the Chinese Society for Agricultural Machinery, 2016, 47(2: 22-27, 34.

[2] Zhou Linyu. Analysis on Pressure Fluctuation and vibration of a centrifugal pump for off-design conditions [J]. Fluid Machinery, 2015(2): 52-55,6.

[3] Zhang Chixiang, Li Yi, Zhang Yuliang. Numerical analysis for inner flow field in a centrifugal pump under maximum operating conditions [J]. Mechanical \& Electrical Engineering Magazine, 2014(8): 974-978.

[4] Liu Houlin, Ren Yun, Wang Kai, et al. Research of inner flow in a double blades pump based on openfoam[J].Journal of Hydrodynamics, 2012, 24(2): 226-234.

[5] Yang Guolai, Wu Guoguo, Liu Xiaoxiong, et al. Dynamic characteristics of axial flow check valve $[\mathrm{J}]$. Journal of Drainage and Irrigation Machinery Engineering, 2013, 31(1): 46-49.

[6] Zheng Shujuan, Quan Long, Chen Qing. Analysis and CFD simulation of the flow filed in a moving poppet valve [J]. Transactions of the Chinese Society for Agricultural Machinery, 2007, 38(1): 168-172.

[7] A. Menéndez Blanco, J.M. Fernández Oro. Unsteady numerical simulation of an air-operated piston pump for lubricating greases using dynamic meshes[J]. Computers and Fluids, 2012, 57: 138-150.

[8] R Castilla, P.J. Gamez-Montero, N Ertürk, et al. Numerical simulation of turbulent flow in the suction chamber of a gear pump using deforming mesh and mesh replacement[J]. International Journal of Mechanical Sciences, 2010, 52: 1334-1342.

[9] Huang Jian, Liu Zhexia, Zhang Lifen, et al. Numerical investigation of the influence of end clearance on the performance of gear pump [J]. Machine Tool \& Hydraulics, 2011, 39(13): 36-38. 